\title{
Pandora of Ebola virus: are we ready?
}

\author{
Ruifu Yang
}

Received: 3 August 2014/Accepted: 3 August 2014/Published online: 27 August 2014

(C) Science China Press and Springer-Verlag Berlin Heidelberg 2014

On 23 March 2014, the ministry of health of Guinea notified WHO of a rapidly spreading outbreak of Ebola virus disease (EVD) in south-eastern forested areas, and 4 days later, the disease was confirmed to be transmitted to Conakry, the Capital of Guinea. The disease also rapidly spread to Liberia, Nigeria and Sierra Leone. As of 27 July 2014, the cumulative number of confirmed (909), probable (276) and suspect (138) EVD cases in the above four countries reached 1,323, including 729 deaths (with the death rate of $55.1 \%$ ) (http://www.who.int/csr/don/2014_ 07_31_ebola/en/). This is the largest outbreak of EVD until now and the first time the disease detected in West Africa.

EVD, previously known as Ebola haemorrhagic fever, is a severe, often fatal illness, with very high case fatality rate of up to $90 \%$. The previous EVD outbreaks in Uganda (epidemic from October of 2000 to February of 2001) and in Gabon and the Republic of the Congo (outbreak from December of 2001 to March of 2002) were also persisted for several months. The outbreak of EVD in West Africa seemed out of control although WHO has coordinated with different organizations and countries for containing this disease [1]. For the last four months, the EVD epidemic has spread to the nearby countries and new cases and new deaths were reported almost every day. Until 6 August 2014, there were 14 outbreaks of EVD and three single case reports in the world, resulting in 4,178 cases of infection and 2,564 deaths, with death rate from $41 \%-88 \%$ (excluding the single case report, Fig. 1).

Ebola was first reported in 1976 when there were outbreaks in Sudan and Democratic Republic of Congo [2, 3].

R. Yang $(\bowtie)$

Beijing Institute of Microbiology and Epidemiology,

Beijing 100071, China

e-mail: ruifuyang@gmail.com
The genus Ebolavirus belongs to the Filoviridae family (filovirus), which also includes genus Marburgvirus and genus Cuevavirus. There are five distinct species in the genus Ebolavirus, including Bundibugyo ebolavirus; Zaire ebolavirus; Sudan ebolavirus; Reston ebolavirus; and Tai Forest ebolavirus. The first three species have been associated with large EVD outbreaks in Africa, whereas the latter two have not. Samples taken from patients of this outbreak have been confirmed to be caused by a strain of ebolavirus closely related $(98 \%)$ to the Zaire one. The ebolavirus should be operated under biosafety level IV laboratory. Even in this high-level containment laboratory, there were still accidentally laboratory-acquired infections reported $[4,5]$.

Fruit bats are believed to be the natural hosts of Ebola virus [6]. The wild animals (monkeys or apes) could be contracted the virus from contacting with bat saliva or faeces. Humans could be transmitted from contacting with infected bats or wild animals. The EVD can be transmitted from person to person by direct contact. More and more evidence showed that pigs might be the host of ebolavirus [7, 8]. From the distribution of fruit bats (http:// www.who.int/csr/disease/ebola/en/\#), the potential risk region of EVD not only is limited to Africa but also includes wide range of America, southern Asia and part of Australia.

Since there is no effective antiviral drugs and specific vaccine for treating and preventing EVD, we all need to prepare for early recognition of EVD and special preventive countermeasures should be taken for the suspected patients. Rapid and specific screening assays should be developed for early diagnosis of EVD; however, the clinical doctors should pay close attention to the suspected patients, especially for those with travel history in the epidemic regions. Hospitals need to prepare to protect 


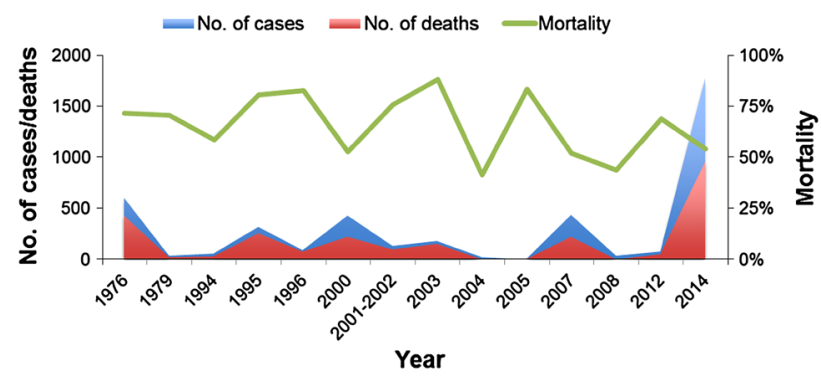

Fig. 1 Summary of EVD outbreaks until August 6, 2014. The figure shows the number of reported cases of EVD and that of deaths. Only one case was reported in 1977, 1988 and 2011, therefore, these years were excluded from the figure to avoid the biased data presentation.

doctors and nurses from ebolavirus infection by contacting the suspected patients. A rapid and effective response should be trained for both professionals in Center for Disease Control and Prevention (CDC) and healthcare personnel. Since there is no border for infectious diseases, every nation should not stand by with folded arms.

WHO has responded quickly by coordinating different countries and organization during EVD outbreak this year and played critical roles in controlling this disease. However, for coping with the emerging infectious diseases, each nation should have a strategic plan to translate relevant basic research into practice. Extensive researches on ebolavirus have been conducted to understand its antigen epitopes and find antiviral targets, which help us greatly in developing diagnostic assays, vaccines and antiviral therapeutics $[9,10]$.

\section{References}

1. Green A (2014) West Africa struggles to contain Ebola outbreak. Lancet 383:1196

2. Report of a WHO/International Study Team (1978) Ebola haemorrhagic fever in Sudan, 1976. Bull World Health Organ $56: 247-270$

3. Report of an International Commission (1978) Ebola haemorrhagic fever in Zaire, 1976. Bull World Health Organ 56:271-293

4. Kortepeter MG, Martin JW, Rusnak JM, Cieslak TJ et al (2008) Managing potential laboratory exposure to ebola virus by using a patient biocontainment care unit. Emerg Infect Dis 14:881-887

5 Gunther S, Feldmann H, Geisbert TW et al (2011) Management of accidental exposure to Ebola virus in the biosafety level 4 laboratory, Hamburg, Germany. J Infect Dis 204:S785-S790

6. Smith I, Wang LF (2013) Bats and their virome: an important source of emerging viruses capable of infecting humans. Curr Opin Virol 3:84-91

7. Pan Y, Zhang W, Cui L et al (2014) Reston virus in domestic pigs in China. Arch Virol 159:1129-1132

8. Weingartl HM, Embury-Hyatt C, Nfon C et al (2012) Transmission of Ebola virus from pigs to non-human primates. Sci Rep 2:811

9. Becquart P, Mahlakoiv T, Nkoghe D et al (2014) Identification of continuous human B-cell epitopes in the VP35, VP40, nucleoprotein and glycoprotein of Ebola virus. PloS one 9:e96360

10. Changula K, Yoshida R, Noyori O et al (2013) Mapping of conserved and species-specific antibody epitopes on the Ebola virus nucleoprotein. Virus res 176:83-90 